\section{Signet-ring cells in the skin: a case of late-onset cutaneous metastasis of gastric carcinoma and a brief review of histological approach}

\author{
Özgür Gündüz, ${ }^{1}$ Mehmet Can Emeksiz, ${ }^{2}$ \\ Pınar Atasoy, ${ }^{3}$ Mehtap Kıdır, ${ }^{4}$ \\ Selim Yalçın, ${ }^{5}$ Serkan Demirkan ${ }^{1}$ \\ 'Department of Dermatology and \\ Venerology, Kırıkkale University; \\ 2Department of Dermatology, Özel \\ Keçiören Hospital; ${ }^{3}$ Department of \\ Pathology Kırıkkale University; \\ ${ }^{4}$ Department of Dermatology and \\ Venerology, Dumlupınar University, \\ Evliya Çelebi Teaching Hospital; \\ ${ }^{5}$ Department of Oncology, Kırıkkale \\ University, Turkey
}

\section{Abstract}

Up to $10 \%$ of patients with visceral malignancies develop skin metastases during their clinical course and these metastases constitute about $2 \%$ of all skin cancers. Skin metastasis may be the first sign of a clinically silent visceral cancer or represent recurrence of an internal malignancy. In both situations, they are associated with poor prognosis, which can partly be attributed to underdiagnosis. In this paper, a case of relapsing gastric adenocarcinoma, which manifested itself as asymptomatic cutaneous papules and nodules on a patient's head and neck, is reported and histopathological approach to the cutaneous lesions containing signet-ring cell is briefly reviewed.

\section{Introduction}

Metastatic skin cancers (MSC) are relatively rare dermatological malignancies. ${ }^{1}$ They constitute $2 \%$ of all skin tumors and reported incidence rates differ from $0.7 \%$ to $9.0 \% .^{1,2}$ MSC originate most commonly from breast, lung and gastrointestinal tissues, and are recognized as a poor prognostic factor. ${ }^{2}$ In this paper, we present a case of relapsing gastric adenocarcinoma with signet-ring cell (SRC) morphology, which presented itself as cutaneous nodular lesions about 4 months after the cessation of chemotherapy.

\section{Case Report}

A 57-year-old woman presented with a 2- month history of asymptomatic, steadily growing lesions on her face, neck and shoulders. Her medical history revealed that she was diagnosed with stage IIIa $\left(\mathrm{T}_{3} \mathrm{~N}_{2} \mathrm{M}_{0}\right)$ gastric $\mathrm{SRC}$ adenocarcinoma (SRCC) 12 months prior in an oncology clinic. At that time, she had underwent total gastrectomy and splenectomy. Her surgical pathology reports revealed cancer-free margins and surgery was followed by adjuvant chemotherapy [infusional folinic acid +5 -Fluorouracil (FUFA)] and radiotherapy directed to the gastric lodge. About four months later after the end of the combined chemotherapy and radiotherapy, the patient had noticed several small lumps on her face and shoulders. Since then, these lesions had been consistently growing. At the time of physical examination, multiple asymptomatic skin lesions consisting of two erythematous papules on her forehead, one solitary nodule on the right preauricular area, another one on her right shoulder and two prominent bright red, fleshy nodules on her neck ranging from $1.0 \times 1.0$ to $3.0 \times 4.0 \mathrm{~cm}$ (Figure 1a-c) were found. An incisonal biopsy was performed from the papule located on the frontal hairline. Histopathological sections showed diffuse dermal infiltration of cells with signet-ring morphology. Immunohistochemical staining were negative for HMB45, CD45, ER, PR, GCDFP-15 stains, lowering the possiblity of an melanoma, a metastatic tumor of hematopoietic origin and a metastatic breast cancer, respectively- and positive for AE1/AE3 and mucicarmine indicating a glandular epithelial origin for the SRCs (Figure 2). Since the patient was operated in another clinic, original resection material was unavailable to us for a comperative histological and immunohistochemical staining. A working diagnosis for metastatic signet-ring cell carcinoma was made on the basis of the last skin biopsises and patient's medical history, and the patient was referred to the oncology department for further evaluation. Tests performed by the oncology department, including complete blood count, urea and electrolytes, liver function tests, plasma proteins, tumor markers, chest X-ray, breast, abdominal and suprapelvic ultrasound, bilateral mammography, complete abdominal magnetic resonance imaging, bilateral mammography, upper gastrointestinal endoscopy and colonoscopy, revealed no other internal focus, from which the skin lesions could originate. After the completion of the oncological examination and tests, diagnosis of the patient was established as metastatic cutaneous signet-ring cell carcinoma and she was scheduled for a second round of chemotherapy (Figure 1d-f).

\section{Discussion}

Signet-ring pattern defines a specific cell shape change, during which the nucleus of the
Correspondence: Özgür Gündüz, Kirikkale Universitesi Tip Fakültesi, Deri ve Zührevi Hastalıkları AD, Yenisehir Mah., Tahsin Duru Cad., No:14, Yahsihan, Kirikkale, Turkey. Tel.: +90.4404071.5408 - Fax: +90.318.2444697. E-mail: gunduzozgur@windowslive.com

Key words: Signet-ring cell, metastatic skin cancer, immunohistochemistry.

Contributions: the authors contributed equally.

Conflict of interest: the authors declare no potential conflict of interest.

Received for publication: 4 August 2016. Accepted for publication: 24 November 2016

This work is licensed under a Creative Commons Attribution-NonCommercial 4.0 International License (CC BY-NC 4.0).

(C) Copyright Ö. Gündüz et al., 2016

Licensee PAGEPress, Italy

Dermatology Reports 2016; 8:6819

doi:10.4081/dr.2016.6819

cell is pushed to the periphery due to the cytoplasmic accumulation of mucin, vacuoles or inclusion bodies, causing the cell to bear a resemblance of a signet-ring. Although SRCs are usually associated with malignancies (i.e., gastric, prostatic, breast cancers, melanoma, etc), ${ }^{3}$ and accepted as a poor prognostic factor, they can also be observed in various nonneoplastic conditions (e.g., pseudomembranous colitis, cystic fibrosis, ulcerative colitis) ${ }^{4}$ or after formalin fixation as a cytoplasmic shrinkage artifact. ${ }^{5}$ In cases without an apparent source for cutaneous SRCs, first step in the differential diagnosis should be to determine whether the cells are neoplastic or not. Wang et al. indicated that even in the setting of a previously diagnosed nonneoplastic disease, benign SRC change can be easily confused with malignant transformation and proposed that lack of the infiltration of lamina propria, rare mitosis and apoptosis and certain histochemical and immunohistochemical staining patterns (positive for E-cadherin and negative for p53 and Ki-67) may be in favor of a nonneoplastic origin for the said SRCs. ${ }^{4}$ Other studies about SRC pathogenesis have shown that malign SRCs posses particular qualities, which may be helpful in developing new diagnostic techniques and treatment modalities. Kobayashi et al. found out that highly differentiated colon adenocarcinoma cells were transformed into SRCs, when phosphatidylinositol 3-kinase (PI3K) was activated. ${ }^{6}$ They also showed that the activation of PI3K was induced by the activation of ErbB2/ErbB3 complex (a member of cell surface receptor family for epidermal growth factor). ${ }^{7}$ At the further steps of 
the ErbB2/ErbB3 - PI3K cascade, p38 mitogen activated protein kinase (MAPK) is activated, resulting in the loss of intercellular tight junctions. ${ }^{8}$ Activation of PI3K is also known to enhance the mucin secretion (Muc4), ${ }^{9}$ and transcriptional upregulation of Muc4 is recognized to have antiapopitotic and mitogenic effects in various tumors. ${ }^{10,11}$ PIK3 activation and Muc4 overexpression have also been found in gastric adenocarcinomas,,$^{12,13}$ which further supports the role of ErbB2/ErbB3 PI3K cascade activation in the formation of mucin-laden neoplastic SRCs.

As mentioned above, reported incidence rates of metastatic skin cancers from internal malignancies vary between $0.7-9.0 \%{ }^{2}$ A metaanalysis including 22,297 patients with visceral malignancies by Krathen et al. ${ }^{14}$ revealed the incidence of skin metastases as 5.3\%. According to this study, the most common primary visceral cancers to metastasize to skin were breast cancers (24.0\%), renal cancers (4.0\%), ovarian cancers (3.8\%), bladder cancers $(3.6 \%)$, lung cancers $(3.4 \%)$, colorectal cancers $(3.4 \%)$ and prostate cancers $(0.7 \%)$ respectively. ${ }^{14}$ Gastric cancers, despite being one of the most common cancers in the world, (ranking 5th in worldwide frequency and 3th in the leading causes of cancer relateddeaths), ${ }^{15}$ do not develop skin metastases frequently. Most common sites for metastatic spread of the gastric cancers were found to be liver, peritoneoum, lung and bone. ${ }^{16}$ Rare skin metastases of gastric cancers are usually observed as umbilical or periumbilical papules or nodules. ${ }^{2}$ Signet-ring cell carcinoma (SRCC) is a histologic subtype of gastric cancers which accounts for $8-30 \%$ of all stomach cancers and is usually associated with advanced stage cancer and recognized as poor prog- nostic factor. ${ }^{14}$ SRCC has a similar metastatic spread pattern like the other gastric cancers and tends to metastasize particularly to peritoneum and intraabdominal organs (liver, ovaries, etc.). Cutaneous metastases of SRCC are also very rare and may present as nodular lesions, such as in this case, mimick benign other nonneoplastic or benign entities such as erysipelas, ${ }^{17}$ scars ${ }^{18}$ or contact dermatitis, ${ }^{19}$ complicating the diagnostic process in the absence of an known primary tumor. Due to their mucin
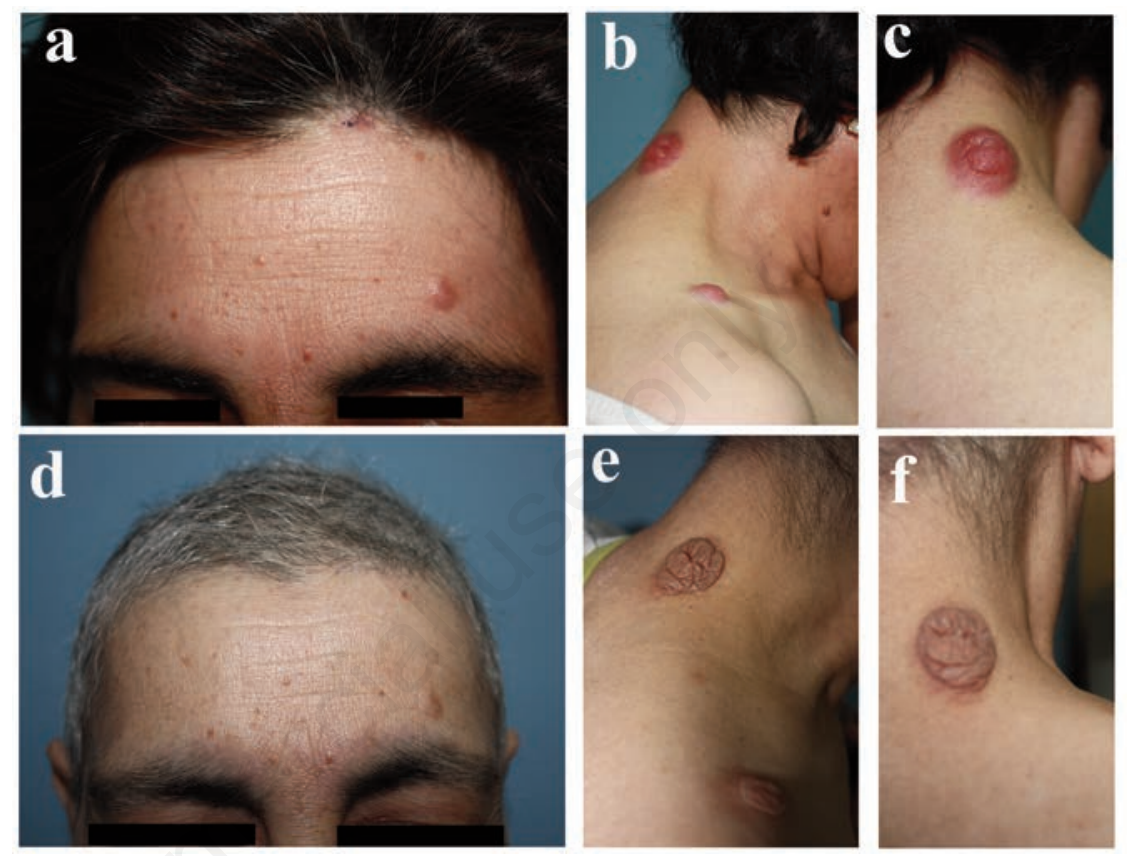

Figure 1. (a,b,c) Numerous asymptomatic fleshy appearing papules and nodules with an elastic texture on the forehead, neck and right shoulder of the patient $(d, e, f)$ Shriveling of the skin nodules, 3 months later after the start of dosetaxel + cisplatin + infusional 5Fluorouracil chemotherapy.

Table 1. Practical histological and immunohistochemical stains for the differential diagnosis of cutaneous signet-ring cells of unknown origin.

Origin of the malign signet-ring cells Histopathological and immunohistochemical clues

Stomach

Colon and rectum

Prostate

Breast

Lung

Intracytoplasmic mucin $(+)$

Stains positive for mucicarmine and AE1/AE3, Alcian Blue, EMA

Intracytoplasmic mucin (+)

Stains positive for mucicarmine, AE1/AE3,CK20, CDX2, MUC2, MUC5AC

Intracytoplasmic mucin (+)

Stains positive PSA, AE1/AE3, mucicarmine, Alcian blue

Intracytoplasmic mucin (+)

Stains positive for MUC1, ER, PR, GCDFP-15, CK7, CEA

Intracytoplasmic mucin (+)

TTF-1, CK7, NapsinA, Mucicarmine, EMA, CEA

AE1/AE3: Immunohistochemical stain which detects CK1-8, 10, 14-16 and 19, in adenocarcinomas Alcian-Blue: Common stain for mucin CDX2: Immunohistochemical stain for a nuclear homeobox transcription factor that belongs to the caudal-related family of CDX homeobox genes and considered specific for enterocytes CEA (Carcinoembriyogenic antigen): found in the normal fetal epithelial cells, also can be detected in metastatic adenocarcinomas. CK (Cytokeratin) 7: Cytokeratin of nonkeratinizing epithelia (breast, lung) CK20: Epithelial marker (positive staining in colon, small intestine and stomach) EMA (Epithelial membrane antigen): Expressed frequently in adenocarcinomas and menengiomas ER (Estrogen Receptor): Immunohistochemical stain for estrogen receptor, relatively specific for breast cancer, nuclear staining (+) GCDFP15 (Gross cystic disease fluid protein-15, Prolactin-induced protein): can be stained immunohistochemically and is considered as a marker of benign and malignant apocrine metaplasia, usually positive in lobular carcinomas with signet ring cells MUC (Mucin): Mucins are a family of high molecular weight, glycosylated proteins produced by epithelial tissues. There are two main families of MUC genes: 1) Genes encoding gel forming /secreted mucins (MUC2, MUC5AC, MUC5B, MUC6) 2) Genes coding membrane bound mucins (MUC1, MUC3A, MUC3B, MUC4, MUC12, MUC13, MUC17) Mucicarmine: Very specific stain for epithelial mucins and adenocarcinomas Napsin-A: Napsin-A is a protein that in humans is encoded by the NAPSA gene and expressed in lung and kidneys. PR (Progesterone receptor): can be identified by immunohistochemical staining and is relatively specific for metastatic tumors from breast. PSA (Prostate specific Antigen, kallikrein-3): Glycoprotein enzyme secreted by the epithelial cells of the prostate gland. Serum levels are elevated in benign and malign prostate diseases. TTF (Thyroid Transcription Factor) 1: is a protein which is encoded by the NKX2-1 gene and regulates transcription of genes particularly in thyroid and lungs. 
required in the differential diagnosis. Immunohistochemical staining can be particularly helpful, if malignant cells of the primary tumor have conserved adequate level of differentiation, These cells will continue to synthesize tissue specific proteins, which can be identified by immunohistochemcial staining [i.e., Thyroid transcription factor (TFF-1) is heavily expressed in lungs and Gross cystic disease fluid protein-15 (GCDFP-15) may be found in well-differentiated breast cancers]. Identification of the tumoral cytokeratin and mucin expression profiles may also help particularly to distinguish adenocarcinomas [i.e., Mucin5A (MUC5A), MUC2 and cytokeratin 20 (CK20) are commonly expressed in colorectal carcinomas, MUC1 and CK7 can be found in breast cancers] (Table 1).

\section{Conclusions}

Signet-ring cell may be seen in various neoplastic and nonneoplastic diseases, but these uncommon cells are frequently associated with malignancies and usually develop due to increased intracellular mucin content. When SRCs are found in a skin biopsy, a high level of suspicion for a cancer should be aroused. Since skin metastases can be observed as the first sign of a clinically-silent malignancy, a detailed medical history should be taken and a thorough physical examination must be conducted for a possible tumor. Appropriate tests should be ordered considering the age and sex specific risk factors for the patient. In case of a cancer of unknown primary origin, histological and immunohistological staining methods would be useful to determine the primary tumor. In very rare instances, when the common histological staining methods are inadequate to distinguish a benign signet-ring change from a malign signet-ring cell transformation, observation invasion of lamina propria and a high intracellular PI3K activity may favor a malign cellular transformation. Actually, MAPK and PI3K signaling pathways are now considered as possible therapeutic targets in various cancers and various methods including Western blotting, phospho-flow cytometry and immunofluorescence staining have been proposed for measuring MAPK and PI3K activity. ${ }^{20}$ Unfortunately, there isn't yet an established and universally accepted method and the proposed methods require specific laboratory equipment and medical laboratory technicians with certain qualifications, preventing the widespread routine use. For similar reasons, we also could not measure the PI3K activity in our specimen. But, despite these facts, MAPK and $\mathrm{PI} 3 \mathrm{~K}$ pathways seem to be as promising targets for future diagnostic and therapeutic interventions.
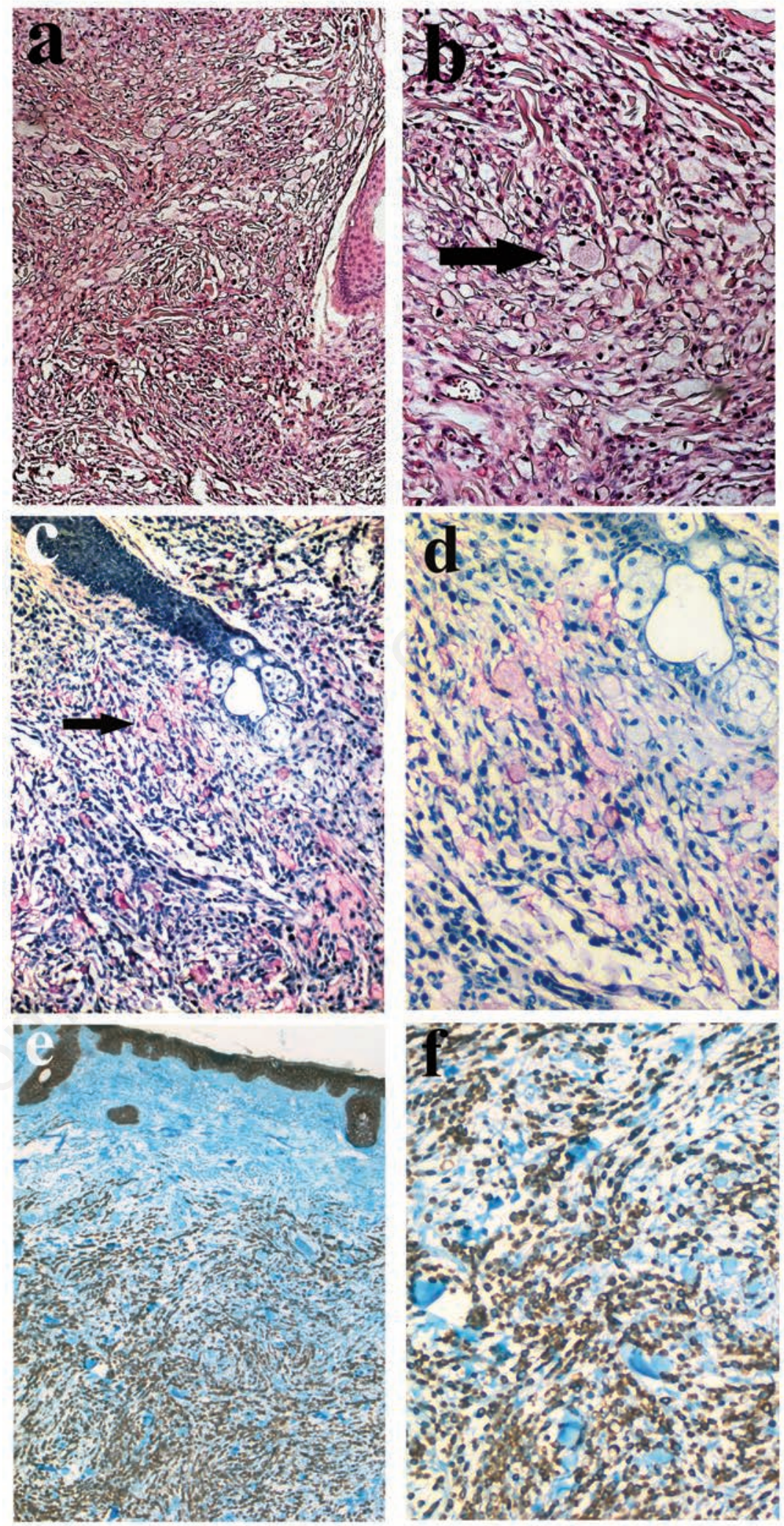

Figure 2. Serial histological sections of the biopsy specimen. a) Signet-ring cells among an eosinophilic dermal infiltrate. Part of an hair follicle can be seen on the right $(\mathrm{H} \& \mathrm{E}$, 100x) b) A typical cell with signet-ring morphology (arrow) (H\&E, 200x) c) Cellular mucin content in the cells near a pilosebeaceous unit indicating glandular origin of epithelial cells (mucicarmine, 100x) d) A close-up of signet-ring cells stained positive for mucin (mucicarmine, 200x) e) Positive staining for cytokeratin indicating an epidermal or epithelial origin (AE1/AE3, 40x) f) SRCs (positive for pancytokeratin) in higher magnification (AE1/AE3, 100x). 


\section{References}

1. Nashan D, Müler ML, Braun-Falco M, et al. Cutaneous metastases of visceral tumours: a review. J Cancer Res Clin Oncol 2009;135:1-14.

2. Hu SC, Chen GS, Lu YW, et al. Cutaneous metastases from different internal malignancies: a clinical and prognostic appraisal. J Eur Acad Dermatol 2008;22:735-40.

3. Fukui Y. Mechanisms behind signet ring cell carcinoma formation. Biochem Biophys Res Commun 2014;450:1231-3.

4. Wang K, Weinrach D, Lal Aseem, et al. Signet-ring cell change versus signet-ring cell carcinoma: a comperative analysis. Am J Surg Pathol 2003;27:1429-33.

5. Arista-Nasr J, Romero-Lagarza P, Pichardo-Bahena R. Artifactual signetring-like cells in endoscopic biopsy of gastric lymphoma. Arch Pathol Lab Med 1997;121:623-5.

6. Kobayashi M, Nagata S, Iwasaki T, et al. Dedifferentiation of adenocarcinomas by activation of phosphatidylinositol 3-kinase. Proc Natl Acad Sci USA 1999 27;96:4874-9.

7. Kobayashi M, Iwamatsu A, ShinoharaKanda A, et al, Activation of ErbB3-PI3kinase pathway is correlated with malignant phenotypes of adenocarcinomas. Oncogene 2003;22:1294-301.
8. Xu Q, Karouji Y, Kobayashi M, et al. The PI3-kinase- Rac-p38 MAP kinase pathway is involved in the formation of signet-ring cell carcinoma. Oncogene 2003;22:553744.

9. El Homsi M, Ducroc R, Claustre J, et al. Leptin modulates the expression of secreted and membrane-associated mucins in colonic epithelial cells by targeting PKC, PI3K, and MAPK pathways. Am J Physiol Gastrointest Liver Physiol 2007;293:G36573.

10. Zhang JJ, Zhu Y, Xie KL, et al. Yin Yang-1 suppresses invasion and metastasis of pancreatic ductal adenocarcinoma by downregulating MMP10 in a MUC4/ErbB2/p38/MEF2C-dependent mechanism. Mol Cancer 2014;13:130.

11. Miyahara N, Shoda J, Kawamoto T, et al. Interaction of Muc4 and ErbB2 in a transgenic mouse model of gallbladder carcinoma: potential pathobiological implications. Oncol Rep 2014;32:1796-802.

12. Tapia 0 , Riquelme $I$, Leal $P$, et al The PI3K/AKT/mTOR pathway is activated in gastric cancer with potential prognostic and predictive significance. Virchows Arch 2014;465:25-33.

13. Tamura Y, Higashi M, Kitamoto S, et al. MUC4 and MUC1 expression in adenocarcinoma of the stomach correlates with vessel invasion and lymph node metastasis: an immunohistochemical study of early gastric cancer. PLoS One 2012;7:e49251.

14. Krathen RA, Orengo IF, Rosen T. Cutaneous metastasis: a meta-analysis of data. South Med J 2003;96:164-7.

15. Pernot S, Voron T, Perkins G, et al. Signetring cell carcinoma of the stomach: impact on prognosis and specific therapeutic challenge. World J Gastroenterol 2015;21: 11428-38.

16. Riihimäki M, Hemminki A, Sundquist K, et al. Metastatic spread in patients with gastric cancer. Oncotarget DOI:10.18632/ oncotarget.10740

17. Müller CSL, Pföhler C, Reichrath J, Tilgen W. Erysipelas carcinomatosum der Abdominalhaut. Hautarzt 2008;59:992-4.

18. Aneiros-Fernandez J, Husein-ElAhmed H, Arias-Santiago S, et al Cutaneous metastasis as first clinical manifestation of signet ring cell gastric carcinoma. Dermatol Online J 2010;16:9.

19. Ahn SJ, Oh SH, Chang SE, et al. Cutaneous metastasis of gastric signet ring cell carcinoma masquerading as allergic contact dermatitis. J Eur Acad Dermatol Venereol 2007;21:123-4.

20. Paraiso KH, Van Der Kooi K, Messina JL, Smalley KS. Measurement of constitutive MAPK and PI3K/AKT signaling activity in human cancer cell lines. Methods Enzymol 2010;484:549-67. 